\title{
Compressed Sensing of Sparse Multipath MIMO Channels with Superimposed Training Sequence
}

\author{
*Bilal Amin, §Babar Mansoor, §Syed Junaid Nawaz, \\ ${ }^{\dagger}$ Shree K. Sharma and ${ }^{\ddagger}$ Mohmammad N. Patwary. \\ *Department of Electrical Engineering, COMSATS Institute of Information Technology, \\ Lahore, Pakistan. \\ ${ }^{\S}$ Department of Electrical Engineering, COMSATS Institute of Information Technology, \\ Islamabad, Pakistan. \\ ${ }^{\dagger}$ SnT - securityandtrust.lu, University of Luxembourg, Luxembourg \\ ${ }^{\ddagger}$ Faculty of Computing Engineering and Sciences, Staffordshire University, Stoke-on-trent, UK. \\ Email: bilalamin@ciitlahore.edu.pk, babar_mansoor@comsats.edu.pk,junaidnawaz@ieee.org, \\ shree.sharma@uni.lu, and m.n.patwary@staffs.ac.uk.
}

\begin{abstract}
Recent advances in multiple-input multiple-output (MIMO) systems have renewed the interests of researchers to further explore this area for addressing various dynamic challenges of emerging radio communication networks. Various measurement campaigns reported recently in the literature show that physical multipath MIMO channels exhibit sparse impulse response structure in various outdoor radio propagation environments. Therefore, a comprehensive physical description of sparse multipath MIMO channels is presented in first part of this paper. Superimposing a training sequence (low power, periodic) over the information sequence offers an improvement in the spectral efficiency by avoiding the use of dedicated time/frequency slots for the training sequence, which is unlike the traditional schemes. The main contribution of this paper includes three superimposed training (SiT) sequence based channel estimation techniques for sparse multipath MIMO channels. The proposed techniques exploit the compressed sensing (CS) theory and prior available knowledge of channel's sparsity. The proposed sparse MIMO channel estimation techniques are named as, SiT based compressed channel sensing (SiT-CCS), SiT based hardlimit thresholding with CCS (SiT-ThCCS), and SiT training based match pursuit (SiT-MP). Bit error rate (BER) and normalized channel mean square error (NCMSE) are used as metrics for the simulation analysis to gauge the performance of proposed techniques. A comparison of the proposed schemes with a notable first order statistics based SiT least squares (SiT-LS) estimation technique is presented to establish the improvements achieved by the proposed schemes. For sparse multipath time-invariant MIMO communication channels, it is observed that SiT-CCS, SiT-MP, and SiT-ThCCS can provide an improvement up to $2 \mathrm{~dB}, 3.5 \mathrm{~dB}$, and $5.2 \mathrm{~dB}$ in the MSE at signal to noise ratio (SNR) of $12 \mathrm{~dB}$ when compared to SiT-LS, respectively. Moreover, for BER $=10^{-1.9}$, the proposed SiT-CCS, SiT-MP, and SiT-ThCCS, compared to SiT-LS, can offer a gain of about $1 \mathrm{~dB}$, $2.5 \mathrm{~dB}$, and $3.5 \mathrm{~dB}$ in the SNR, respectively. The performance gain in MSE and BER is observed to improve with an increase in the channel sparsity.
\end{abstract}

Keywords: MIMO, superimposed training, first-order statistics, compressed sensing, channel estimation

Corresponding author: Syed Junaid Nawaz, Department of Electrical Engineering, Academic Block-I, COMSATS Institute of Information Technology (CIIT), Chak-Shahzad, Park Road, Islamabad, 44000, Pakistan. Mob: +92 300 9119472, Email: junaidnawaz@ieee.org.

The partial contents of this paper were presented in International Conference on Telecommunications (ICT), Thessalonilki, May 2016 [1]. 


\section{INTRODUCTION}

The channel impulse response (CIR) of several outdoor radio propagation environments tends to be sparse in nature [2-4]. A particular cellular communication environment with distant dominant scatterers, as shown in Fig. 1a exhibits a sparse CIR [4]. In aeronautical communication channels, as illustrated in Fig. 1b, we not only have a line-of-sight (LoS) path but also a cluster of scattered multipath components due to reflection from large scattering objects. Therefore, impulse response of an aeronautical communication channel is sparse in nature. The wideband high frequency (HF) communication channel is also sparse in time domain due to long delay spread and very fewer multipath components [5], as shown in Fig. 1c. The sparse impulse response is also exhibited in underwater acoustic communication channels [6], as depicted in Fig. 1d. Similarly, in high-definition television (HDTV) broadcast scenario, as shown in Fig. 1e, there are only a few dominant echoes but the CIR comprises of manifolds of symbol duration $[7,8]$. Such sparse propagation channels have only certain dominant multipath components that are largely separated in delay domain, which makes channel estimation a challenging task [9].

In the literature, several sparse channel estimation techniques have been proposed - see e.g., [10-16]. In [16], authors have established the fact that during estimation of sparse channels, use of mean squared error (MSE) criterion along with $\ell_{1}$-norm outperforms the Wiener filter results and conventional estimation methods. In [11], the authors have proposed a matching pursuit (MP) algorithm in order to estimate a sparse channel. In [13], orthogonal matching pursuit (OMP) algorithm have been proposed to overcome the convergence issues of MP algorithm. In [14], compressed channel sensing (CCS) theory has been used for sparse channel estimation that

exploits sparsity of the channel and outperforms the conventional least squares based methods. In [12], the authors have proposed sparse cognitive matching pursuit (SCMP) algorithm for the estimation of sparse channel for MIMO orthogonal frequency division multiplexing (MIMOOFDM). Furthermore, the authors claim that SCMP requires no prior knowledge of the channel sparsity in order to obtain an accurate estimate of CIR. In conventional training based channel estimation approaches, a known training sequence is multiplexed with the information sequence in time, frequency, and/or code domain at the transmitter, and the receiver estimates the channel 


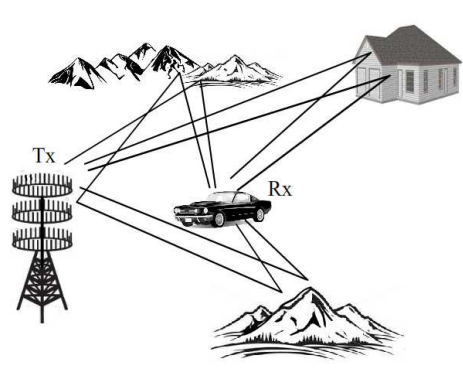

(a)

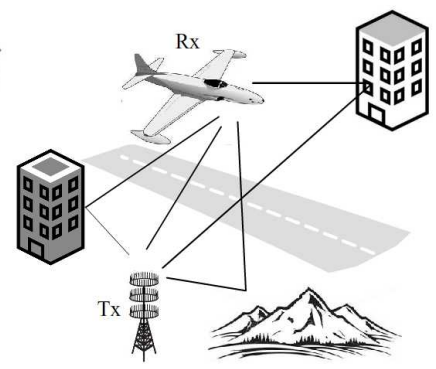

(b)

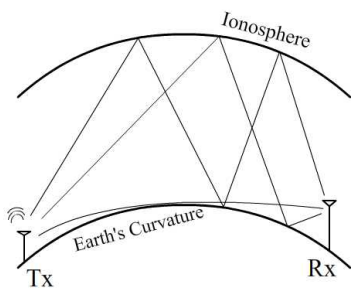

(c)

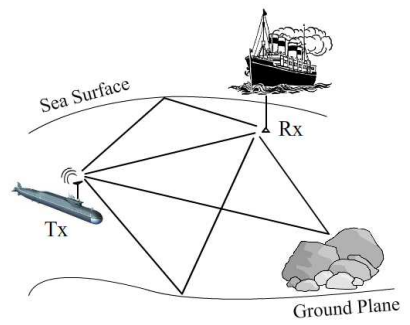

(d)

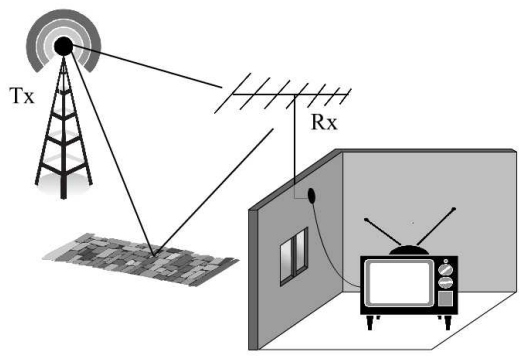

(e)

Fig. 1: Sparse multipath channel propagation environments. (a) Land cellular communications, (b)Aeronautical communications, (c) HF communications, (d) Underwater acoustic communication, (e) Terrestrial television broadcast

by exploiting this known training sequence and its corresponding received signal. This scheme imposes an overhead on the spectral efficiency of the system. In blind channel estimation techniques, the receiver explicitly estimates the channel by only using the known statistical properties of the transmitted information sequence, thus, avoiding any overhead of training sequence. However, in case of blind channel estimation, long data sequences are needed at the receiver resulting in slow convergence [17]. In superimposed training based (SiT) channel estimation methods, a known training sequence is superimposed over the data sequence. This avoids overhead on speed by preventing any use of dedicated time/frequency slots for training sequence [18]. SiT based techniques are not only spectrally efficient but also effectively track the channel variations. An SiT based technique was first proposed by Frahang Boroujeny in [19] for single antenna systems. In [20], a channel estimation technique, based on first order statistics of the information sequence, has been proposed for single-input multiple-output (SIMO) time-invariant channels. In [18], authors have proposed a SiT sequence based approach for time invariant MIMO channels. For the estimation of sparse underwater acoustic channels, a SiT 
based channel estimation technique is proposed in [9]. In [21], a genetic algorithms (GA) based sparse multipath channels estimation technique with SiT sequence has been presented. In [4], authors have proposed a compressed sampling based technique for sensing of sparse multipath channels with SiT for single-input single-output (SISO) systems.

Large scale MIMO systems are thought to be a potential candidate to address various dynamic challenges of fifth generation (5G) communication networks [22]. This has, thus, renewed the interest of the researchers in the MIMO systems. Various recently conducted outdoor measurement campaigns show that physical multipath MIMO channels exhibit sparse impulse response structure. Therefore, it is now highly desirable to develop channel estimation techniques for sparse MIMO channels. To the best of authors' knowledge, no such technique for the estimation of sparse MIMO communication channels with SiT sequence is available in the literature. Nevertheless, this paper thus proposes SiT based compressive channel sensing techniques for time invariant sparse MIMO channels. This paper first presents a new analytical model for sparse MIMO channels in Section II. The considered communication system model is presented in Section III. The proposed SiT based sparse MIMO channel estimation techniques are presented in Section IV. The simulation results along with a comprehensive performance analysis of the proposed techniques is presented in Section V. Section VI presents the conclusion.

Notations: Boldface uppercase italic letters, e.g., $\boldsymbol{H}$ represent matrices. Boldface lowercase italic letters represent vectors, e.g., $\boldsymbol{h}$. Scalar quantities are denoted with small case italic letters, e.g., $h$. Hermitian transpose of a vector is represented as [.]*.

\section{PhysicAl CHANNEL MOdEL FOR MIMO Systems}

The proposed physical model for sparse MIMO communication channels is illustrated in Fig. 2. The transmitter and receiver antenna arrays consist of $N$ and $M$ antenna elements, respectively. The horizontal and vertical orientation of both the antenna arrays are modeled as flexible to be independently rotatable. The transmitter and receiver antenna arrays are considered as mobile with velocity $v_{T} \mathrm{~m} / \mathrm{s}$ and $v_{R} \mathrm{~m} / \mathrm{s}$ and the direction of their motion as $\theta_{v_{T}}$ and $\theta_{v_{R}}$, respectively. The adjacent antenna elements in both the arrays are taken as equally separated by a distance $d_{\lambda}$. 


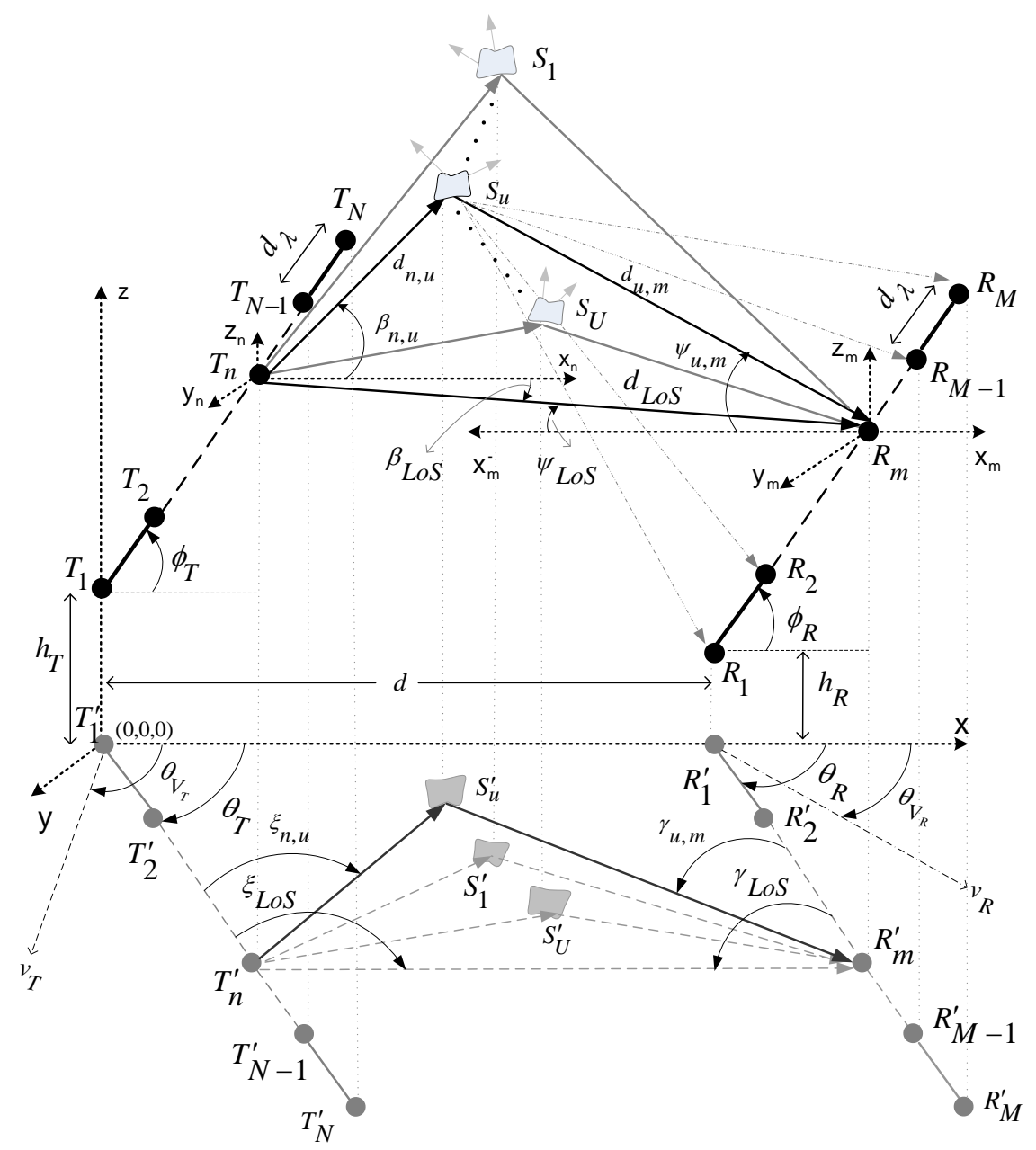

Fig. 2: Physical model for sparse MIMO communication channels.

The origin of coordinate system is assumed at the base of first element of the transmit antenna array. The coordinates of the first elements of the transmit and receive antenna arrays can thus be represented by $\left(0,0, h_{T}\right)$ and $\left(d, 0, h_{R}\right)$, respectively. The elevation of transmitter and receiver arrays is denoted by $h_{T}$ and $h_{R}$, respectively. Subsequently, the coordinates of the $n^{\text {th }}$ transmitter antenna element can be obtained as below,

$$
\begin{aligned}
& x_{n}=(n-1) d_{\lambda} \cos \phi_{T} \cos \theta_{T}, \\
& y_{n}=(n-1) d_{\lambda} \cos \phi_{T} \sin \theta_{T}, \\
& z_{n}=(n-1) d_{\lambda} \sin \phi_{T}+h_{T} .
\end{aligned}
$$

Similarly, the coordinates of the $m^{\text {th }}$ receiver antenna element can be obtained as under, 


$$
\begin{aligned}
& x_{m}=(m-1) d_{\lambda} \cos \phi_{R} \cos \theta_{R}+d, \\
& y_{m}=(m-1) d_{\lambda} \cos \phi_{R} \sin \theta_{R} \\
& z_{m}=(m-1) d_{\lambda} \sin \phi_{R}+h_{R} .
\end{aligned}
$$

where $\theta_{T}$ and $\phi_{T}$ represent the rotation angles of transmitter antenna array in azimuth and elevation planes, respectively. Similarly, $\theta_{R}$ and $\phi_{R}$ are the angles of rotation of receiver antenna array in azimuth and elevation planes, respectively.

The distances from $n^{\text {th }}$ transmitter and $m^{\text {th }}$ receiver antenna element to a $u^{\text {th }}$ scattering object are denoted by $d_{n, u}$ and $d_{u, m}$, respectively. These distances can be expressed in generalized form as below,

$$
\begin{aligned}
& d_{n, u}=\sqrt{\left(x_{u}-x_{n}\right)^{2}+\left(y_{u}-y_{n}\right)^{2}+\left(z_{u}-z_{n}\right)^{2}}, \\
& d_{u, m}=\sqrt{\left(x_{n}-x_{u}\right)^{2}+\left(y_{n}-y_{u}\right)^{2}+\left(z_{n}-z_{u}\right)^{2}},
\end{aligned}
$$

where the coordinates of a $u^{t h}$ arbitrary scattering object are denoted by $\left(x_{u}, y_{u}, z_{u}\right)$. For simulation of the proposed channel model, the coordinates of scattering objects may be drawn from a certain distribution within the defined entire scattering region or a subregion [23]. The number of scattering objects, within a defined region, may be drawn from a Poisson distribution [23]. The distribution and the number of scattering objects can be tuned according to the available empirical measurements. Various distinct types of distributions have been used in the literature for modelling the physical location of scattering objects in different types of propagation environment, e.g., uniform [24], Gaussian [25,26], and hyperbolic [26], etc. The length of multipath corresponding to $u^{t h}$ scattering object can be calculated as, $d_{n, u, m}=d_{n, u}+d_{u, m}$. The length of LoS path from $n^{\text {th }}$ transmitter to $m^{\text {th }}$ receiver is given as follows,

$$
d_{n, m}=\sqrt{\left(x_{m}-x_{n}\right)^{2}+\left(y_{m}-y_{n}\right)^{2}+\left(z_{m}-z_{n}\right)^{2}} .
$$

Azimuth and elevation angles of departure (AoD) from the $n^{\text {th }}$ transmitter to the $u^{\text {th }}$ scatterer are denoted by $\xi_{n, u}$ and $\beta_{n, u}$, respectively. These angles can be expressed as follows, 


$$
\begin{gathered}
\xi_{n, u}=\pi-\arctan \left(\frac{y_{u}-y_{n}}{x_{u}-x_{n}}\right)-\theta_{T} \\
\beta_{n, u}=\arctan \left(\frac{z_{u}}{x_{u}}\right) .
\end{gathered}
$$

Similarly, azimuth and elevation angles of arrival (AoA) from $u^{\text {th }}$ scatterer to the $m^{\text {th }}$ receiver antenna element are represented by $\gamma_{u, m}$ and $\psi_{u, m}$, respectively; and are given as below,

$$
\begin{gathered}
\gamma_{u, m}=\pi-\arctan \left(\frac{y_{m}-y_{u}}{x_{m}-x_{u}}\right)-\theta_{R}, \\
\psi_{u, m}=\arctan \left(\frac{z_{u}}{x_{m}-x_{u}}\right) .
\end{gathered}
$$

The azimuth and elevation angles formed at $n^{\text {th }}$ transmitter element along the LoS direction towards $m^{\text {th }}$ receiver element are denoted by $\xi_{n, m}$ and $\beta_{n, m}$, respectively; which can be obtained as below,

$$
\begin{gathered}
\xi_{n, m}=\pi-\arctan \left(\frac{y_{m}-y_{n}}{x_{m}-x_{n}}\right)-\theta_{T}, \\
\beta_{n, m}=\arctan \left(\frac{z_{m}-z_{n}}{d_{L o S}}\right) .
\end{gathered}
$$

Similarly, the LoS angles formed at the receiver side can be expressed as below,

$$
\begin{gathered}
\gamma_{n, m}=\pi-\arctan \left(\frac{y_{m}-y_{n}}{x_{m}-x_{n}}\right)-\theta_{R}, \\
\psi_{n, m}=\arctan \left(\frac{z_{m}-z_{n}}{x_{m}-x_{n}}\right) .
\end{gathered}
$$

In the case of no mobility, the delay $\tau_{n, u, m}$ of a certain propagation path from $n^{\text {th }}$ transmitter to $m^{\text {th }}$ receiver associated with $u^{\text {th }}$ scatterer, can be found as below,

$$
\tau_{n, u, m}=\frac{d_{n, u, m}}{c}
$$

where $c$ represents the velocity of electromagnetic waves' propagation, i.e., $c=3 \times 10^{8} \mathrm{~m} / \mathrm{s}$. The minimum path delay for the channel between $n^{t h}$ transmitter and $m^{\text {th }}$ receiver element is 
exhibited by the LoS path, and is given by,

$$
\tau_{\min }=\frac{d_{L o S}}{c}
$$

while the maximum path delay $\tau_{\max }$ is exhibited by the scatterer having the longest path. In the proposed channel model, both the ends of communication link are taken as mobile, which imposes time variability in the channel characteristics. The length of a certain propagation path, from $n^{\text {th }}$ transmitter to $m^{\text {th }}$ receiver corresponding to $u^{\text {th }}$ scattering object, thus changes with time, depending upon the direction and velocity of mobility. The length of a portion of the multipath, i.e., from $n^{\text {th }}$ transmitter to $u^{\text {th }}$ scatterer, can be expressed as,

$$
\dot{d}_{n, u, m}(t)=\sqrt{2 d_{n, u}^{2}+v_{T}^{2} t^{2}-2 v_{T} t d_{n, u} \cos \beta_{n, u} \cos \left(\theta_{T}+\xi_{n, u}-\theta_{v_{T}}\right)} .
$$

Similarly, the length of the other portion of the multipath, i.e., from $u^{t h}$ scatterer to the $m^{\text {th }}$ receiver, can be obtained as below,

$$
\dot{d}_{u, m}(t)=\sqrt{2 d_{u, m}^{2}+v_{R}^{2} t^{2}-2 v_{R} t d_{u, m} \cos \psi_{u, m} \cos \left(\theta_{R}+\gamma_{u, m}-\theta_{v_{R}}\right)} .
$$

Thus, the total path length from the $n^{\text {th }}$ transmitter to the $m^{\text {th }}$ receiver after observation time of $t$ seconds becomes as, $\dot{d}_{n, u, m}(t)=\dot{d}_{n, u}(t)+\dot{d}_{u, m}(t)$. Therefore, the path delay associated with $\dot{d}_{n, u, m}(t)$ can be found as below,

$$
\dot{\tau}_{n, u, m}(t)=\frac{\dot{d}_{n, u, m}(t)}{c}
$$

The time-variant impulse response of the multipath fading channel, comprising of $U$ paths, between $n^{\text {th }}$ transmitter and $m^{\text {th }}$ receiver, can be written as,

$$
h_{n m}(\tau ; t)=\sum_{u=1}^{U} \chi_{u}(t) \mathrm{e}^{-j 2 \pi f_{c} \dot{\tau}_{n, u, m}(t)} \delta\left(\tau-\dot{\tau}_{n, u, m}(t)\right),
$$

where $f_{c}$ is the carrier frequency and $\delta($.$) denotes the standard Kronecker delta function. The$ attenuation factor associated with $u^{t h}$ path is represented by $\chi_{u}(t)$, such that, $E\left\{\sum_{u}\left|\chi_{u}\right|^{2}\right\}=1$. The channel impulse response $h_{n m}(\tau ; t)$ is a complex Gaussian random process with respect 
to time $t$. For the scenario when the scattering environment also has certain fixed contributing scattering objects (or LoS component), the envelope $|h(\tau ; t)|$ has a Rice distribution. When the differential path delay is smaller than a symbol duration, the channel exhibits a flat response in frequency domain and channel impulse response given in (19) can be written independent of $\tau[23,27]$. The path delay is modeled as a multiple of the symbol duration and the total number of resolvable propagation paths is denoted by $L$, such that $U \geq L$. Moreover, when the communication nodes are static, the channel exhibits a time independent behaviour and impulse response vector in (19) can be written independent of $t$.

As discussed earlier, in various realistic propagation environments, when the scattering environment has only a few largely distant dominant scattering objects, the channel exhibits a sparse impulse response. In such scenario, when the channel impulse response vector from $n^{\text {th }}$ transmitter to $m^{t h}$ receiver has only $Q$ non-zero values at delay positions $\breve{\boldsymbol{p}}_{n m}=\left[\breve{p}_{0}, \breve{p}_{1}, \cdots, \breve{p}_{Q-1}\right]$. The sparse channel impulse response vector can thus be represented as,

$$
\boldsymbol{h}_{n m}^{l}= \begin{cases}\neq 0 & ; l \in \breve{\boldsymbol{p}}_{n m} \\ =0 & ; \text { otherwise }\end{cases}
$$

The impulse response vector of a channel is said to be $Q$ sparse, if $\left\{Q=\left\|\boldsymbol{h}_{n m}\right\|_{\ell_{0}}\right\} \ll L$. For MIMO systems where the separation among transmitter antennas and receiver antennas (i.e., $d_{\lambda}$ ) is a fraction of the distance travelled by an electromagnetic wave within a symbol duration, the channel support may only differ by an unresolvable amount of delay among the channels between adjacent elements of antenna arrays [28]. For such scenario, the support vectors $\breve{\boldsymbol{P}}_{n m}$ may be same for all the values of $n$ and $m$. The channel impulse response vector from $n^{\text {th }}$ transmitter to $m^{t h}$ receiver can be written as, $\boldsymbol{h}_{n m}=\left[h_{n m}^{0}, h_{n m}^{1}, \cdots, h_{n m}^{L-1}\right]^{*}$. MIMO channel matrix for a certain delay $l$ can be written as,

$$
\boldsymbol{H}^{l}=\left[\begin{array}{cccc}
h_{11}^{l} & h_{12}^{l} & \cdots & h_{1 N}^{l} \\
h_{21}^{l} & h_{22}^{l} & \cdots & h_{2 N}^{l} \\
\vdots & \cdots & \ddots & \vdots \\
h_{M 1}^{l} & h_{M 2}^{l} & \cdots & h_{M N}^{l}
\end{array}\right]
$$


MIMO channel convolutional matrix can be expressed as,

$$
\boldsymbol{H}=\left[\begin{array}{cccc}
\boldsymbol{H}^{0} & \cdots & \boldsymbol{H}^{L-1} & \mathbf{0} \\
\vdots & \ddots & \ddots & \vdots \\
\mathbf{0} & \boldsymbol{H}^{0} & \cdots & \boldsymbol{H}^{L-1}
\end{array}\right]
$$

\section{Proposed Communication Model For MiMO Systems}

The proposed MIMO communication system model has a block diagram as shown in Fig. 3. Let $N$ and $M$ be the number of transmit and receive antenna array elements, respectively. The signal transmitted from a transmit antenna propagates through a sparse MIMO communication channel with $Q$ non-zero taps. Channel estimator (CE) is implemented with various first-order statistics based techniques, which are; SiT based compressed channel sensing (SiT-CCS), SiT based hardlimit thresholding with CCS (SiT-ThCCS), and SiT training based match pursuit (SiT-MP). The training-sequence effect remover (TER) eliminates the contribution of training sequence after estimation of the channel's impulse response and feeds the equalizer with a regularized version of the received signal. A linear minimum mean square equalizer (LMMSE) is implemented to estimate the information sequence. Let $\boldsymbol{b}_{n}=\left[b_{n}(0), b_{n}(1), \cdots, b_{n}(K-1)\right]^{*}$ represent zero-mean information sequence such that $\boldsymbol{b}_{n}$ is mutually independent for each of the $n^{\text {th }}$ user. A known deterministic and periodic training sequence $\boldsymbol{c}_{n}=\left[c_{n}(0), c_{n}(1), \cdots, c_{n}(K-\right.$ 1) $]^{*}$, having period $P$ such that $c_{n}(k)=c_{n}(k+a P)$ for $k$ and $a$ be any integers, is superimposed (arithmetically added) over the information sequence $\boldsymbol{b}_{\boldsymbol{n}}$. The superimposed information and training sequences for a specific $n^{\text {th }}$ transmitter is given as below,

$$
\boldsymbol{x}_{n}=\boldsymbol{b}_{n}+\boldsymbol{c}_{n}
$$

The sequence $\boldsymbol{x}_{n}=\left[x_{n}(0), x_{n}(1), \cdots, x_{n}(K-1)\right]^{*}$ is transmitted over the jointly sparse MIMO channel such that the impulse response between $n^{\text {th }}$ transmitter and $m^{\text {th }}$ receiver is given by $\boldsymbol{h}_{n m}=\left[h_{n m}^{0}, h_{n m}^{1}, \cdots, h_{n m}^{L-1}\right]^{*}$, where $L$ is length of the channel. The signal received at time instant $k$ by the $m^{\text {th }}$ antenna element of receiver array is given by the following equation, 


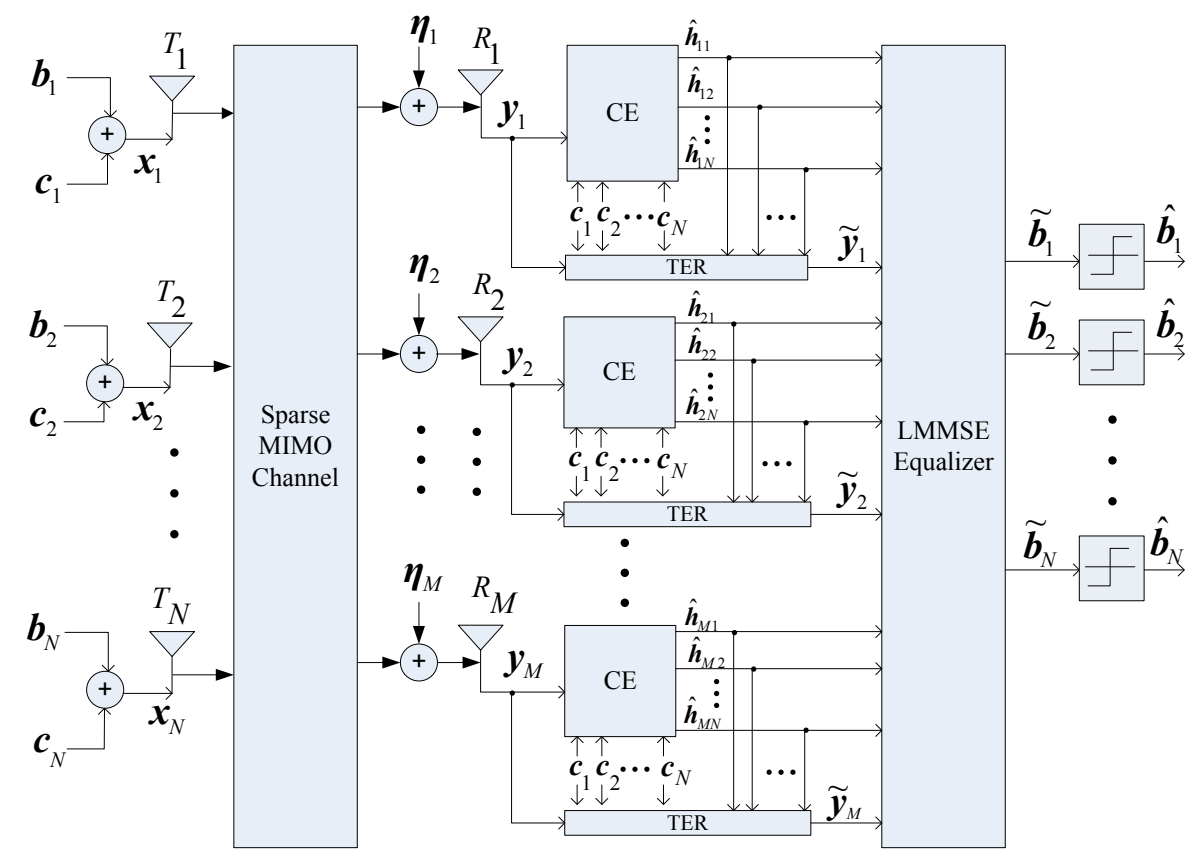

Fig. 3: Block Diagram of the Proposed MIMO Communication System.

$$
y_{m}(k)=\sum_{n=1}^{N} \sum_{l=0}^{L-1} h_{n m}^{l} x_{n}(k-l)+\eta_{m}(k),
$$

where $\eta_{m}(k)$ denotes $k^{\text {th }}$ sample of zero mean complex-valued additive white Gaussian noise (AWGN) with variance $\sigma_{\eta}^{2}$. The signal received by all the antenna elements of receiver at time instant $k$ is $\boldsymbol{y}(k)=\left[y_{1}(k), y_{2}(k), \cdots, y_{M}(k)\right]^{*}$ and given by,

$$
\boldsymbol{y}(k)=\sum_{l=0}^{L-1} \boldsymbol{H}^{l} \boldsymbol{x}(k-l)+\boldsymbol{\eta}(k),
$$

where $\boldsymbol{x}(k-l)=\left[x_{1}(k-l), x_{2}(k-l), \cdots, x_{N}(k-l)\right]^{*}$ and $\boldsymbol{\eta}(k)=\left[\eta_{1}(k), \eta_{2}(k), \cdots, \eta_{M}(k)\right]^{*}$. Temporal sampling yields following representation of the received signal,

$$
\breve{\boldsymbol{y}}(k)=\boldsymbol{H} \breve{\boldsymbol{x}}(k)+\breve{\boldsymbol{\eta}}(k),
$$

where

$$
\begin{aligned}
\breve{\boldsymbol{y}}(k) & =\left[\boldsymbol{y}^{*}(k+L-1), \boldsymbol{y}^{*}(k+L-2), \cdots, \boldsymbol{y}^{*}(k)\right]^{*}, \\
\breve{\boldsymbol{x}}(k) & =\left[\boldsymbol{x}^{*}(k+L-1), \boldsymbol{x}^{*}(k+L-2), \cdots, \boldsymbol{x}^{*}(k)\right]^{*}, \\
\breve{\boldsymbol{\eta}}(k) & =\left[\boldsymbol{\eta}^{*}(k+L-1), \boldsymbol{\eta}^{*}(k+L-2), \cdots, \boldsymbol{\eta}^{*}(k)\right]^{*} .
\end{aligned}
$$




\section{MIMO CHANNEL ESTIMATION BASED ON FIRST-ORDER STATISTICS WITH SIT.}

For a given MIMO communication system if each user is assigned with a specific training sequence which is added with the information sequence, then, first order statistics of the received signal can be used to estimate the channel outlined in [20]. In this section, the SiT based channel estimation technique of [18] is extended for the estimation of sparse MIMO channels. In this technique, each user is assigned with a distinct cycle frequency. Suppose for a specific transmitter $n$ the training sequence $c_{n}(k)$ is periodic. The period of the training sequence is $P=\tilde{P} N$, where $\tilde{P}$ is a positive integer. The training sequence $c_{n}(k)$ is given as below,

$$
c_{n}(k)=\sum_{i=0}^{P-1} c_{i, n} \mathrm{e}^{j(2 \pi i / P) k}, \quad \forall k,
$$

where, $j=\sqrt{-1}$ and

$$
c_{i, n}=\frac{1}{P} \sum_{k=0}^{P-1} c_{n}(k) \mathrm{e}^{-j(2 \pi i / P) k},
$$

Choose $c_{n}(k)$ in such a way that only $\tilde{P}$ coefficients out of total $P$ are non zero, so $c_{n}(k)$ can be written as follows,

$$
c_{n}(k)=\sum_{i=0}^{\tilde{P}-1} c_{i, n}^{\prime} \mathrm{e}^{j \alpha_{i, n} k}, \quad \forall k,
$$

where $\alpha_{i, n}=2 \pi(i N+n-1) / P$, and $c_{i, n}^{\prime}$ are suitably chosen coefficients for $1 \leq n \leq N$ and $0 \leq i \leq \tilde{P}-1$. In order to design $c_{n}(k)$, first choose a periodic base sequence $\bar{c}_{o}(k)$ that has a period of $\tilde{P}[18]$ in such a way that,

$$
\bar{c}_{i, o}=\frac{1}{\tilde{P}} \sum_{k=0}^{\tilde{P}-1} \bar{c}_{o}(k) \mathrm{e}^{-j(2 \pi i / \tilde{P}) k} .
$$

The periodic training sequence $\overline{c_{1}}(n)$, with period $P$, is generated by replicating $\bar{c}_{o}(k)$ for $N$ times. The training sequence of a specific transmitter $n$ can, therefore, be defined as follows [18],

$$
c_{n}(k)=\sigma_{c n} \bar{c}_{1}(k) \mathrm{e}^{j(2 \pi / P)(n-1) k} \text { for } n=1,2, \ldots, N .
$$

Expectation of the received signal $y_{m}(k)$ at $m^{\text {th }}$ receiver can be found as below, 


$$
E\left\{y_{m}(k)\right\}=\sum_{n=1}^{N} \sum_{i=0}^{\tilde{P}-1}\left[\sum_{l=0}^{L} c_{i, n}^{\prime} h_{n m}^{l} \mathrm{e}^{-j \alpha_{i, n} l}\right] \mathrm{e}^{j \alpha_{i, n} k}
$$

For $n_{1} \neq n_{2}$, we have $\alpha_{i_{1}, n_{1}} \neq \alpha_{i_{2}, n_{2}}$ for any $\left\{i_{1}, i_{2}\right\} \in 0,1, \ldots, \tilde{P}-1$. Let $\boldsymbol{d}_{n m}=$ $\left[d_{n m, 0}, d_{n m, 1}, \cdots, d_{n m,(\tilde{P}-1)}\right]^{*}$, where $d_{n m, i}$ is given by,

$$
d_{n m, i}=\sum_{l=0}^{L} c_{i, n}^{\prime} h_{n m}^{l} \mathrm{e}^{-j \alpha_{i, n} l}
$$

The mean square consistent estimate $\hat{\boldsymbol{d}}_{n m}=\left[\hat{d}_{n m, 0}, \hat{d}_{n m, 1}, \cdots, \hat{d}_{n m,(\tilde{P}-1)}\right]^{*}$ of $\boldsymbol{d}_{n m}$ can be obtained by computing its coefficient as given in [18], which is as follows,

$$
\hat{d}_{n m, i}=\frac{1}{T} \sum_{k=0}^{T-1} y_{m}(k) \mathrm{e}^{-j \alpha_{i, n} k}
$$

where $T$ represents the number of received symbols, as $T \rightarrow \infty, \hat{d}_{n m, i} \rightarrow d_{n m, i}$. The relationship given in (34) can also be written vector form as below,

$$
\hat{\boldsymbol{d}}_{n m}=\boldsymbol{C}_{n} \boldsymbol{h}_{n m}
$$

where $\boldsymbol{C}_{n}$ can be obtained as

$$
\boldsymbol{C}_{n}=\operatorname{diag}\left\{c_{0, n}^{\prime}, c_{1, n}^{\prime}, \cdots, c_{(\tilde{P}-1), n}^{\prime}\right\} \boldsymbol{V}_{n}
$$

where $\boldsymbol{V}_{n}$ can be found as,

$$
\boldsymbol{V}_{n}=\left[\begin{array}{cccc}
1 & 1 & \cdots & 1 \\
1 & \mathrm{e}^{-j \alpha_{1, n}} & \cdots & \mathrm{e}^{-j \alpha_{1, n} L} \\
1 & \mathrm{e}^{-j \alpha_{2, n}} & \cdots & \mathrm{e}^{-j \alpha_{2, n} L} \\
\vdots & \vdots & \vdots & \vdots \\
1 & \mathrm{e}^{-j \alpha_{(\tilde{P}-1), n}} & \cdots & \mathrm{e}^{-j \alpha_{(\tilde{P}-1), n} L}
\end{array}\right]
$$




\section{A. SiT based least squares (SiT-LS)}

The least squares estimate of the channel between $n^{\text {th }}$ transmitter and $m^{\text {th }}$ receiver can be obtained from the linear model in ((35)), as proposed in [20], and is given below,

$$
\hat{\boldsymbol{h}}_{n m}^{\mathrm{SiT}-\mathrm{LS}}=\arg \min _{\tilde{\boldsymbol{h}}_{n m}}\left\|\hat{\boldsymbol{h}}_{\boldsymbol{n} \boldsymbol{m}}-\boldsymbol{C}_{n} \tilde{\boldsymbol{h}}_{n m}\right\|_{\ell 2}^{2}
$$

The above estimate can also be obtained as,

$$
\hat{\boldsymbol{h}}_{n m}^{\mathrm{SiT}-\mathrm{LS}}=\left(\boldsymbol{C}_{n}^{*} \boldsymbol{C}_{n}\right)^{-1} \boldsymbol{C}_{n}^{*} \hat{\boldsymbol{d}}_{n m}
$$

To obtain the channel estimate for non-zero mean noise, set $\tilde{P} \geq L+1$, omit first row from $\boldsymbol{C}_{n}$ and $\hat{d}_{n m, 0}$ from $\hat{\boldsymbol{d}}_{n m}$.

\section{B. Proposed SiT based MIMO Channel Estimation}

The error in the estimate $\hat{d}_{n m, i}$ of $d_{n m, i}$ can be realized by substituting $y_{m}(k)$ from (24) in (34). The simplified solution for $\hat{d}_{n m, i}$ can be expressed as under,

$$
\hat{d}_{n m, i}=d_{n m, i}+\varepsilon_{n m, i}
$$

where $\varepsilon_{n m, i}$ represents the error in the estimate of $d_{n m, i}$. The estimation error $\varepsilon_{n m, i}$ contains contribution from additive noise $\left(\tilde{\eta}_{n m, i}\right)$, interference from superimposed information sequence of all the transmitters $\left(\tilde{b}_{n m, i}\right)$, and interference from training sequence of cross channels $\left(\tilde{c}_{\tilde{n} m, i}\right)$. The estimation error is thus given by, $\varepsilon_{n m, i}=\tilde{c}_{\tilde{n} m, i}+\tilde{b}_{n m, i}+\tilde{\eta}_{n m, i}$; where,

$$
\begin{gathered}
\tilde{c}_{\tilde{n} m, i}=\frac{1}{T} \sum_{k=0}^{T-1}\left[\sum_{\substack{\tilde{n}=1 \\
\tilde{n} \neq n}}^{N-1} \sum_{l=0}^{L} h_{\tilde{n} m}^{l} c_{\tilde{n}}(k-l)\right] \mathrm{e}^{-j \alpha_{i, \tilde{n}} k}, \\
\tilde{b}_{n m, i}=\frac{1}{T} \sum_{k=0}^{T-1}\left[\sum_{n=1}^{N-1} \sum_{l=0}^{L} h_{n m}^{l} b_{n}(k-l)\right] \mathrm{e}^{-j \alpha_{i, n} k},
\end{gathered}
$$




$$
\tilde{\eta}_{n m, i}=\frac{1}{T} \sum_{k=0}^{T-1} \eta_{m}(k) \mathrm{e}^{-j \alpha_{i, n} k}
$$

Ignoring the inherent error $\varepsilon_{n m, i}$ leads to a non-realistic estimate of the channels. Moreover, the first order statistics based technique presented in previous section is not optimized for the case of sparse multipath channel. This section, thus, presents three extensions of the first-order statistics based MIMO channel estimation model in (35) for the case of sparse multipath channels by incorporating a compensation for the inherent estimation error $\varepsilon_{n m, i}$. The proposed techniques include SiT-CCS, SiT-ThCCS, and SiT-MP.

1) SiT based CCS: During the past few years, compressed sensing has emerged as a new paradigm for sampling and reconstruction of sparse signals. It has been established in [2932] that a finite-dimensional sparse signal can be exactly reconstructed from fewer, linear, and nonadaptive measurements by solving a well-defined convex optimization problem. In literature CS approach has been established as an efficient solution to estimate sparse multipath channels see e.g., [4,33-35]. To ensure exact reconstruction of the received signal, a measurement matrix should satisfy restricted isometry property (RIP), [29,30]. For the linear model given in (35), the condition for RIP is given by,

$$
\left(1-\delta_{Q}\right)\left\|\boldsymbol{h}_{n m}\right\|_{\ell_{2}}^{2} \leq\left\|\boldsymbol{C}_{n} \boldsymbol{h}_{n m}\right\|_{\ell_{2}}^{2} \leq\left(1+\delta_{Q}\right)\left\|\boldsymbol{h}_{n m}\right\|_{\ell_{2}}^{2}
$$

where $0<\delta_{Q}<1$ is the restricted isometry constant of the measurement $\boldsymbol{C}_{n}$. If the condition given in (44) holds then $\boldsymbol{C}_{n}$ satisfies RIP of order $Q$ and is sufficient for exact recovery of the sparse channel. In order to estimate SiT based sequence of MIMO channel, the $\ell_{1}$ minimization problem can be recast from the model in (35), as given below,

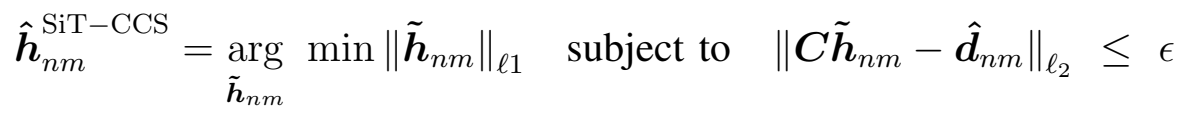

where the parameter $\epsilon$ is proportional to magnitude of the error $\varepsilon_{n m}$, i.e., $\epsilon \propto\left\|\varepsilon_{n m}\right\|_{\ell_{2}}^{2}$. The convex optimization problem given in (45) can be solved using a compressed sensing based technique known as Dantzig selector (DS) [36]. The DS performs near optimal with high computational 
efficiency and improved recovery accuracy.

2) SiT based Hard-limit Thresholded CCS: The estimated channel impulse response, obtained by using CCS, consists of some nominal non-zero values, whereas, the correct estimate of the taps is a zero value. Hard limiting on the estimated channel impulse response vector according to a predefined threshold $(\zeta)$ level ensures a sparse vector. Such hard-limit thresholding can be applied as follows,

$$
\hat{h}_{n m}^{l, \mathrm{SiT}-\mathrm{ThCCS}}= \begin{cases}\hat{h}_{n m}^{l, \mathrm{SiT}-\mathrm{CCS}} & ; \hat{h}_{n m}^{l, \mathrm{SiT}-\mathrm{CCS}}>\zeta \\ 0 & ; \text { otherwise }\end{cases}
$$

where $\hat{h}_{n m}^{l, \mathrm{SiT}-\mathrm{CCS}}$ represents the estimate of $l^{\text {th }}$ tap of the channel from $n^{\text {th }}$ transmitter to $m^{\text {th }}$ receiver obtained by the CCS technique presented in previous section.

3) MP based SiT Sparse Multipath MIMO Channel Estimation : Since the channel under consideration is sparse, a large number of taps in the channel vector $\boldsymbol{h}_{n m}$ is either zero or below the noise floor. Thus, an MP algorithm can be employed to estimate the sparse channel as proposed in [11]. Hence, to estimate the channels from the model in (35), the positions of nonzero taps are first determined and channel estimation is then carried out only for these specific non-zero positions. We describe the proposed SiT-MP algorithm in the following paragraphs. In (35), both $\boldsymbol{C}_{n}$ and $\hat{\boldsymbol{d}}_{n m}$ are known, therefore, $\hat{\boldsymbol{d}}_{n m}$ can be expanded as,

$$
\hat{\boldsymbol{d}}_{n m}=\overline{\boldsymbol{c}}_{n, o} h^{0}+\overline{\boldsymbol{c}}_{n, 1} h^{1}+\overline{\boldsymbol{c}}_{n, 2} h^{2}+\ldots+\overline{\boldsymbol{c}}_{n, \tilde{P}-1} h^{\tilde{P}-1}
$$

where $\overline{\boldsymbol{c}}_{n, i}$ is the $i^{\text {th }}$ column vector of $\boldsymbol{C}_{n}$. First, find columns in matrix $\boldsymbol{C}_{n}=\left[\overline{\boldsymbol{c}}_{n, 0}, \overline{\boldsymbol{c}}_{n, 1}, \ldots, \overline{\boldsymbol{c}}_{n,(\tilde{P}-1)}\right]$ that are best aligned with output vector $\hat{\boldsymbol{d}}_{n m}$; let this vector be denoted by $\overline{\boldsymbol{c}}_{q p}$. Let $Q$ be the number of non-zero taps among a total of $L$ channel taps. The output vector $\hat{\boldsymbol{d}}_{n m}$ is spanned by total $Q$ columns of $\boldsymbol{C}_{n}$ that actually correspond to $Q$ non-zero entries of the sparse channel $\boldsymbol{h}_{n m}$. By projecting all columns of $\boldsymbol{C}_{n}$ on $\hat{\boldsymbol{d}}_{n m}$, we can find the best aligned column of $\boldsymbol{C}_{n}$ with $\hat{\boldsymbol{d}}_{n m}$ that will correspond to the position of one of the non-zero entries of $\boldsymbol{h}_{n m}$. In this way, we can find the location of non-zero entries present in the sparse 
channel $\boldsymbol{h}_{n m}$ in each iteration. Once the non-zero tap position of $\boldsymbol{h}_{n m}$ is determined, the value at that tap position can be found. The algorithm proceeds in the same way for each iteration. In order to find the non-zero tap positions of $\boldsymbol{h}_{n m}$ in the $p^{\text {th }}$ iteration, the projection of $\boldsymbol{C}_{n}$ along $\hat{\boldsymbol{d}}_{n m}$ is found as,

$$
q^{p}=\underset{\overline{\boldsymbol{c}}_{n, j} \neq\left\{\overline{\boldsymbol{c}}_{n, q^{1}}, \ldots, \overline{\boldsymbol{c}}_{n, q^{p-1}}\right\}}{\arg } \max \frac{\left|\overline{\boldsymbol{c}}_{n, j}^{*} \tilde{\boldsymbol{d}}_{\boldsymbol{n} \boldsymbol{m}}^{p-1}\right|^{2}}{\left\|\overline{\boldsymbol{c}}_{n, j}\right\|_{\ell_{2}}^{2}}
$$

where $q_{p}$ represents the index of best aligned column of $\boldsymbol{C}_{n}$ with $\hat{\boldsymbol{d}}_{n m}$ and corresponds to one of the non-zero tap $\boldsymbol{h}_{n m}$. The projection of $\boldsymbol{C}_{n}$ in each iteration is computed along the residual error vector of previous iteration $\tilde{\boldsymbol{d}}_{n m}^{p-1}$. For the very first iteration the residual error vector is $\tilde{\boldsymbol{d}}_{n m}^{o}=\hat{\boldsymbol{d}}_{n m}$ and in the preceding iterations its value is attained as,

$$
\tilde{\boldsymbol{d}}_{n m}^{p}=\tilde{\boldsymbol{d}}_{n m}^{p-1}-\frac{\boldsymbol{c}_{q_{p}}^{*} \tilde{\boldsymbol{d}}_{n m}^{p-1}}{\left\|\boldsymbol{c}_{q^{p}}^{*}\right\|_{\ell_{2}}^{2}} \boldsymbol{c}_{q^{p}}
$$

where $\boldsymbol{c}_{q^{p}}^{*}$ denotes the best aligned column vector of $\boldsymbol{C}_{n}$ with residual error vector $\tilde{\boldsymbol{d}}_{n m}^{p-1}$ for a specific $p^{\text {th }}$ iteration that corresponds to the non-zero entry of sparse channel. The estimate of a non-zero tap of the channel $\hat{h}_{n m}^{q^{p}}$ at position $q^{p}$ can thus be obtained as,

$$
\hat{h}_{n m}^{q^{p}, \mathrm{SiT}-\mathrm{MP}}=\frac{\boldsymbol{c}_{q^{p}}^{*} \tilde{\boldsymbol{d}}_{\boldsymbol{n} \boldsymbol{m}}^{p-1}}{\left\|\boldsymbol{c}_{q^{p}}^{*}\right\|_{\ell_{2}}^{2}} .
$$

The iterations continue until all the non-zero taps in $\hat{\boldsymbol{h}}_{n m}^{\mathrm{SiT}-\mathrm{MP}}$ are determined, or when the error residual in a specific iteration becomes smaller than a predefined threshold, i.e., $\left\|\tilde{\boldsymbol{d}}_{n m}^{p}\right\|<\epsilon$. The cost of computing the basic model presented in equation (35) is same for all the proposed techniques. However, the additional computational cost to estimate the channels from (35) varies for each of the proposed techniques i.e., SiT-LS, SiT-MP, SiT-CCS, and SiT-ThCCS. Each iteration of the matching pursuit algorithm for computing a particular channel's estimate $\hat{\boldsymbol{h}}_{n m}$ implies a cost of $O(\tilde{P} \log (\tilde{P}))$ in addition to the cost of computing (35). The proposed SiT-CCS estimation technique is implemented using Dantzig selector solution to (45). The Dantzig selector implemented by using primal-dual method, which incurs a computational cost of $O\left(\sqrt{\tilde{P}} \log \left(\frac{\tilde{P}}{\epsilon}\right)\right)$. For the SiT-ThCCS technique, the hard-limiting of the obtained channels' 
estimate extends an additional computational cost, proportional to the length of channel $L$, over the cost implied by SiT-CCS.

\section{Minimum Mean Square Error (MMSE) Equalizer}

Since the training sequence assigned to each of the transmitter in MIMO system is also known at their corresponding receivers so we have to cancel out the effect of training sequence that also gets convolved with channel when superimposed with information sequence. Thus before passing the signal to equalizer input we must have to cancel out the effect of convolved training sequence of the receiver whose information sequence is to be determined along with the convolved training sequence of other users as well. Once the effect of training sequence is omitted we can input that signal at equalizers input. The following steps are required to equalize the superimposed training based information sequence,

$$
\tilde{y}_{m}(k)=y_{m}(k)-\sum_{n=1}^{N} \sum_{l=0}^{L} \hat{h}_{n m}^{l} c_{n}(k-l),
$$

where $\hat{h}_{n m}^{l}$ represents the estimate of $l^{\text {th }}$ tap of channel from $n^{\text {th }}$ transmitter to $m^{\text {th }}$ receiver. The estimate of channel may be taken from any of the estimation techniques discussed in the previous sections, i.e., $\hat{\boldsymbol{h}}_{n m}=\left\{\hat{\boldsymbol{h}}_{n m}^{\mathrm{SiT}-\mathrm{LS}}, \hat{\boldsymbol{h}}_{n m}^{\mathrm{SiT}-\mathrm{CCS}}, \hat{\boldsymbol{h}}_{n m}^{\mathrm{SiT}-\mathrm{MP}}\right.$, or $\left.\hat{\boldsymbol{h}}_{n m}^{\mathrm{SiT}-\mathrm{ThCCS}}\right\}$. For the equalizer at the $m^{\text {th }}$ receiver, the optimal equalizer's weights $\boldsymbol{w}_{m}$ can be obtained as in [37], given as under,

$$
\boldsymbol{w}_{n m}=\left.\left(\hat{\boldsymbol{H}} \hat{\boldsymbol{H}}^{*}+2 \sigma_{m}^{2} \boldsymbol{I}\right)^{-1} \hat{\boldsymbol{H}}\right|_{(m-1)\left(L_{e}+L-1\right)+\left(\tau_{d}+1\right)},
$$

where $m$ is the receiver index such that $1 \leq m \leq M, L_{e}$ denotes length of equalizer, $\tau_{d}$ is the decision delay of equalizer's mappers, $\boldsymbol{I}$ denotes the $\left(N \times L_{e}\right) \times\left(N \times L_{e}\right)$ identity matrix and $\left.\hat{\boldsymbol{H}}\right|_{i}$ is the $i^{t h}$ column of $\hat{\boldsymbol{H}}$. The convolutional matrix $\hat{\boldsymbol{H}}$ having dimensions $L_{e} \times\left(L_{e}+L-1\right)$ is given by,

$$
\hat{\boldsymbol{H}}_{n, m}=\left[\begin{array}{ccccccc}
\hat{h}_{n, m}^{0} & \hat{h}_{n, m}^{1} & \cdots & \hat{h}_{n, m}^{L-1} & 0 & \cdots & 0 \\
0 & \hat{h}_{n, m}^{0} & \hat{h}_{n, m}^{1} & \cdots & \hat{h}_{n, m}^{L-1} & \ddots & \vdots \\
\vdots & \ddots & \ddots & \ddots & \ddots & \ddots & 0 \\
0 & \ldots & 0 & \hat{h}_{n, m}^{0} & \hat{h}_{n, m}^{1} & \cdots & \hat{h}_{n, m}^{L-1}
\end{array}\right]
$$


The overall convolutional matrix $\hat{\boldsymbol{H}}$ of the MIMO system is give as,

$$
\hat{\boldsymbol{H}}=\left[\begin{array}{cccc}
\hat{\boldsymbol{H}}_{1,1} & \hat{\boldsymbol{H}}_{1,2} & \cdots & \hat{\boldsymbol{H}}_{1, M} \\
\hat{\boldsymbol{H}}_{2,1} & \boldsymbol{H}_{2,2} & \cdots & \hat{\boldsymbol{H}}_{2, M} \\
\vdots & \vdots & \cdots & \vdots \\
\hat{\boldsymbol{H}}_{N, 1} & \hat{\boldsymbol{H}}_{N, 2} & \cdots & \hat{\boldsymbol{H}}_{N, M}
\end{array}\right]
$$

The estimate of transmitted information sequence from the $n^{t h}$ transmitter can thus be obtained from the following equation followed by a decision mapper, as shown in Fig. 3.

$$
\tilde{b}_{n}(k)=\sum_{m=1}^{M} \sum_{i=0}^{L_{e}-1} w_{n m}^{i} \tilde{y}_{m}(k-i) \text {. }
$$

The output of the equalizer, $\tilde{\boldsymbol{b}}_{n}$, is then fed as an input to a decision mapper, as shown in Fig. 3, that performs mapping of the symbols according to the used modulation scheme with the decoded symbols represented by $\hat{\boldsymbol{b}}_{n}$.

\section{RESUlts AND Discussion}

In this section, we present the computer simulations along with an analysis of the obtained results. The performance metrics used for the analysis are NCMSE and BER, which are well established metrics. The BER quantifies reliability of the radio channel, which can be defined as the ratio between the amount of corrupted bits and total number of transmitted bits. The normalized channel mean square error (NCMSE) of the estimated channel is defined as,

$$
\mathrm{NCMSE}=\frac{\sum_{m=1}^{M} \sum_{n=1}^{N} \sum_{l=0}^{L-1}\left|\hat{h}_{n m}^{l}-h_{n m}^{l}\right|^{2}}{\sum_{m=1}^{M} \sum_{n=1}^{N} \sum_{l=0}^{L-1}\left|h_{n m}^{l}\right|^{2}},
$$

A $2 \times 2$ MIMO communication system is considered for the simulations, i.e., $N=2$ and $M=2$. The simulations are performed for time-invariant and frequency selective MIMO channels. The realization of all the channels $h_{n m}$ is independently generated for each Mote Carlo run, by keeping a certain fixed level of sparsity, $Q / L$. The amount of scatterers within a certain range is drawn from Poison distribution by adapting a sub-region approach discussed in [23], and 
the location of scatterers within each subregion is drawn from uniform distribution. To ensure a certain level of sparsity, all the scatterers except the scatterers corresponding to the selected positions $\left(\breve{\boldsymbol{P}}_{n m}\right)$ of non-zero taps are discarded. However, the support (non-zero delay positions' vectors $\breve{\boldsymbol{P}}_{n m}$ ) of impulse response vector for all the channels is drawn independently from uniform distribution. The values of non-zero taps of a channel $h_{n m}$ follow zero-mean Gaussian distribution. The number of resolvable multipath components are taken equal for all the channels, fixed at $L=14$ with variance $1 /(M(L+1))$. A periodic training sequence is generated following the $m$-sequence approach presented in [18]. A periodic base sequence with period $\tilde{P}=15$ is taken fixed as, $\{-1,-1,-1,1,1,1,1,-1,1,-1,1,1,-1,-1,1\}$, for all the simulation results. Zero mean white Gaussian noise is independently generated at each receiver satisfying a certain signal-tonoise (SNR) ratio. The SNR at $m^{\text {th }}$ receiver is defined as the ratio between variance of received signal $\sigma_{y_{m}}^{2}$ and variance of noise $\sigma_{m}^{2}$, i.e., $\mathrm{SNR}_{m}=\sigma_{y_{m}}^{2} / \sigma_{m}^{2}$. The zero mean BPSK modulated information sequences $\left(\boldsymbol{b}_{n} \in\{1,-1\}\right)$ is generated mutually independent for each transmitter.

Performance comparison of the proposed techniques and the first order statistics based technique (SiT-LS) in [18] is presented in Fig. 4a and Fig. 4b for NCMSE and BER against SNR, respectively. The message length and sparsity level for this plot are taken as $K=900$ bits and $Q / L=3 / 14$, respectively. In Fig. $4 \mathrm{a}$ it can be observed that the proposed channel estimation techniques (i.e., SiT-CCS, SiT-MP, and SiT-ThCCS) outperforms the first order statistics based channel estimation technique (i.e., SiT-LS). When compared to SiT-LS, the proposed techniques SiT-CCS, SiT-MP, and SiT-ThCCS provide an improvement of $2 \mathrm{~dB}, 3.5 \mathrm{~dB}$, and 5.2dB in MSE at an $\mathrm{SNR}=12 d B$, respectively. This improvement in the plots from the proposed techniques is due to the consideration of a compensation parameter for the inherent estimation error $\varepsilon_{n m}$ and use of prior available knowledge of channels' sparsity. The proposed techniques provide, better performance compared to the first order statistics based technique for the case of sparse channels, and similar performance for the case of non-sparse channels. Fig. 4b present BER based comparison of the proposed and SiT-LS estimation techniques. It can be observed that a performance gain of about $1 \mathrm{~dB}, 2.5 \mathrm{~dB}$, and $3.5 \mathrm{~dB}$ in SNR can be achieved by SiT-CCS, SI-MP, and SiT-ThCCS when compared to SiT-LS for BER $=10^{-1.9}$. The BER performance can further 


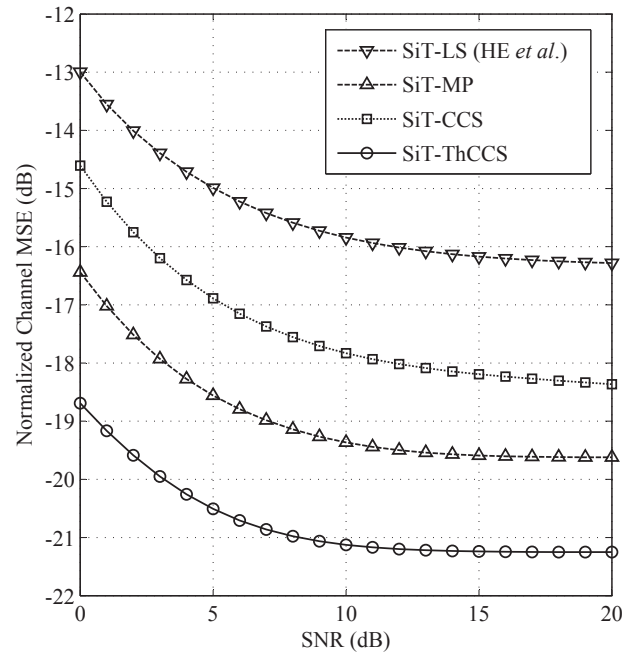

(a) MSE based comparison.

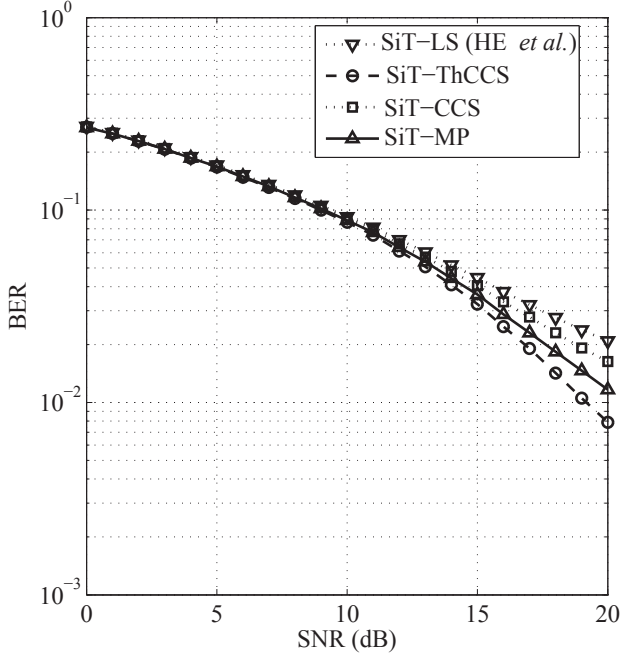

(b) BER based comparison.

Fig. 4: MSE and BER based comparative analysis between SiT-LS and the proposed SiT-CCS, SiT-ThCCS, and SiT-MP for 900 bits message signal length.

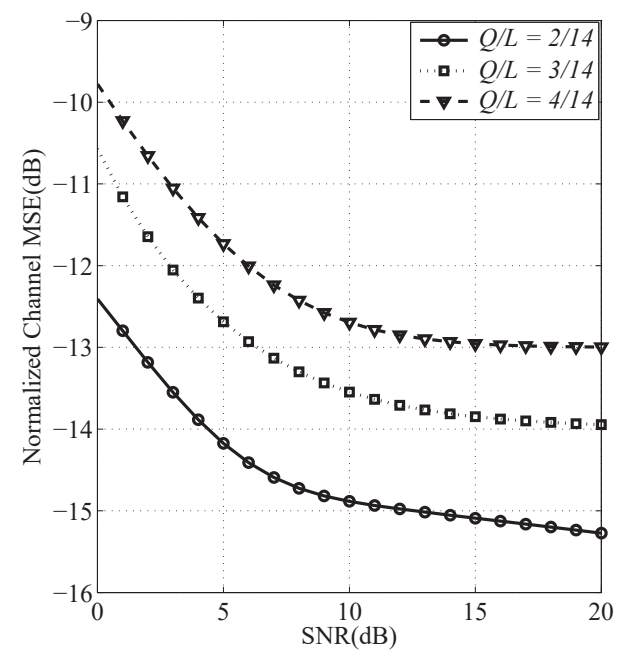

(a) Effect of sparsity level $Q$ on SiT-MP performance.

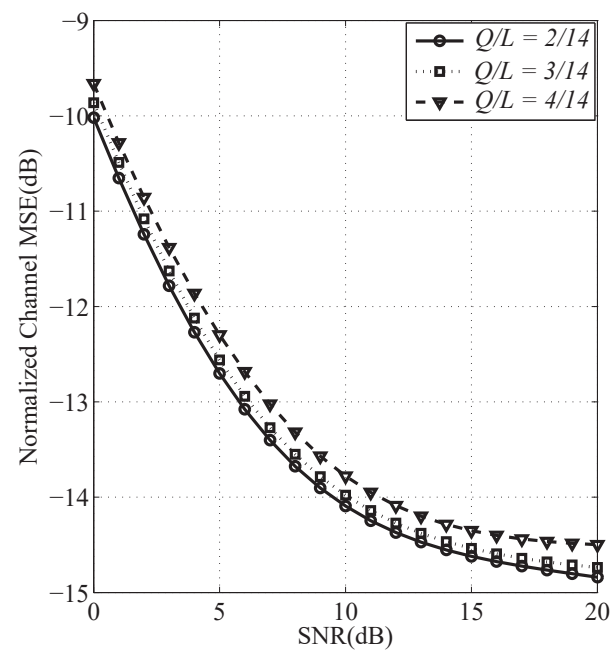

(b) Effect of Sparsity level $Q$ on SiT-CCS performance.

Fig. 5: Effect of variation of sparsity level $Q$ on the MSE Performance of the proposed SiT-MP and SiT-CCS techniques.

be enhanced by employing the iterative algorithm proposed in [4].

To demonstrate the effect of channel's sparsity level (i.e., $Q / L$ ), the MSE is plotted against SNR for different values of sparsity (i.e., $Q / L=2 / 14,3 / 14$, and 4/14) in Fig. 5a and Fig. 5b for the proposed SiT-MP and SiT-CCS, respectively. For these plots, the training to information 


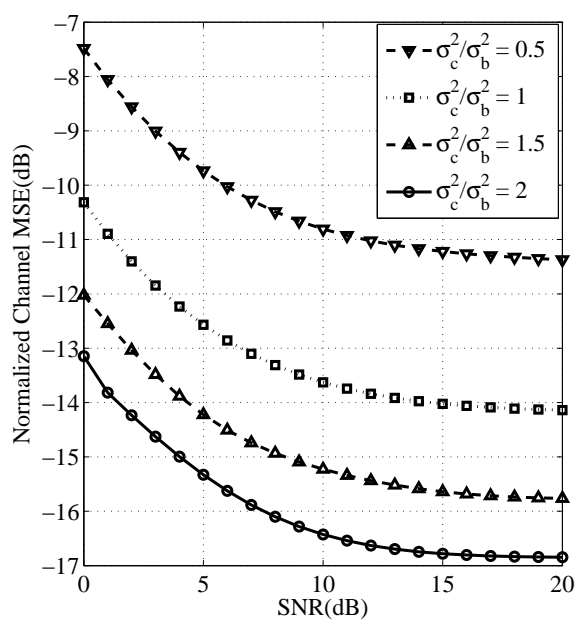

(a) Effect of TIR $\left(\sigma_{c}^{2} / \sigma_{b}^{2}\right)$ on SiT-MP.

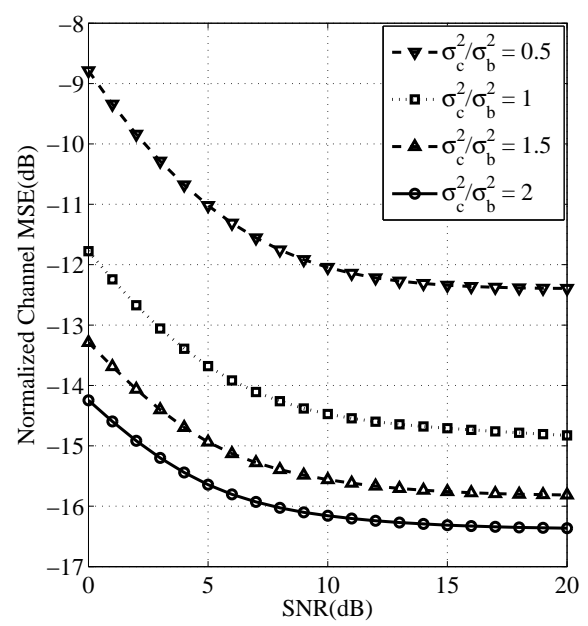

(b) Effect of TIR $\left(\sigma_{c}^{2} / \sigma_{b}^{2}\right)$ on SiT-CCS.

Fig. 6: Effect of the variation of TIR $\left(\sigma_{c}^{2} / \sigma_{b}^{2}\right)$ on MSE performance of the proposed SiT-MP and SiT-CCS techniques.

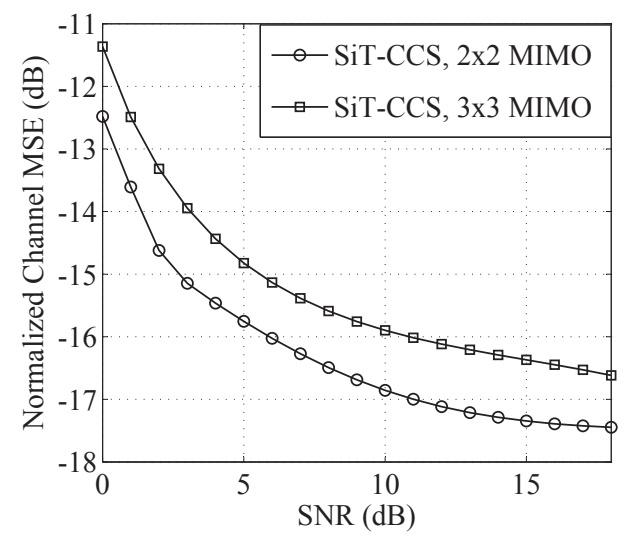

(a) MSE performance of SiT-CCS.

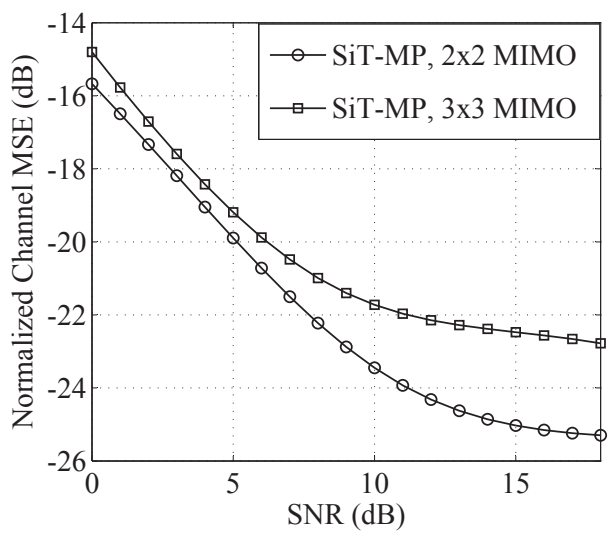

(b) MSE performance of SIT-MP.

Fig. 7: MSE performance comparison of SiT-CCS and SiT-MP for $2 \times 2$ and $3 \times 3$ MIMO system.

sequence power ratio $\sigma_{c}^{2} / \sigma_{b}^{2}$ is set to unity. It can be observed that by decreasing number of non-zero taps, i.e., by decreasing $Q$ for a fixed $L$, the MSE performance improves for all SNR values obtained from both the SiT-CCS and SiT-MP estimation techniques. Thus, the performance of the proposed schemes further improve with an increase in the channel's sparsity. The gain in performance improvement with an increase in the channel's sparsity is higher for SiT-MP technique when compared with the performance gain by SiT-CCS technique. 


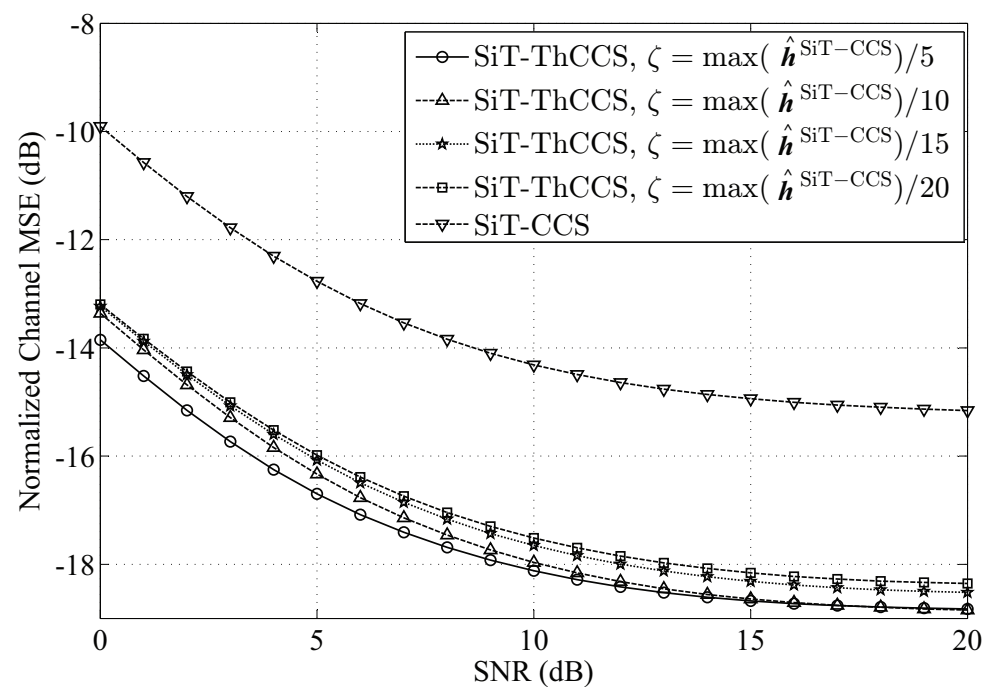

Fig. 8: Effect of hard-limit threshold $\zeta$ on the MSE performance of the proposed SiT-ThCCS technique.

To elucidate the effect of TIR (i.e., $\sigma_{c}^{2} / \sigma_{b}^{2}$ ) on the performance of the proposed SiT-MP and SiT-CCS channel estimation techniques, the MSE is plotted against SNR for different values of TIR, ranging from 0.5 to 2 with uniform difference of 0.5, in Fig. 6a and Fig. 6b, respectively. These graphs are obtained for channel's sparsity level $Q / L$ set to $3 / 14$. It can be noted that with an increase the training sequence's power (i.e., $\sigma_{c}^{2}$ ) for fixed information sequence's power (i.e., $\left.\sigma_{b}^{2}\right)$, the MSE performance of channel estimator is observed to improve. However, decreasing the contribution of information sequence has an adverse effect on the BER performance of the system in decoding of information sequence. Therefore, choice of an optimum value for TIR is highly resaleable to satisfy a good tradeoff between MSE and BER performance.

In order to prove the validity of the proposed channel estimation techniques for higher order MIMO systems, the MSE performance analysis for a $3 \times 3$ MIMO systems have been performed in comparison with a $2 \times 2$ MIMO system in Fig.7. This graph is obtained for the TIR taken as $\sigma_{c}^{2} / \sigma_{b}^{2}=0.4$ and sparsity of the channels set to $Q / L=4$. To demonstrate the effect of hardlimiting threshold parameter, $\zeta$, on the proposed SiT-ThCCS estimation technique, MSE is plotted against SNR for different values of $\zeta$ in Fig. 8. This plot is obtained for the channel's sparsity level $Q / L$ set to $3 / 14$ and TIR $\sigma_{c}^{2} / \sigma_{b}^{2}$ set to unity. The plot is generated for different values 
of limiting threshold, viz: $1 / 5,1 / 10,1 / 15$, and1/20 times the highest value of channel impulse response vector; where, the initial channel estimate is obtained through SiT-CCS technique. It is evident in the plot that MSE performance improves with a decrease in the value of $\zeta$ up to a certain optimum value, and the plot shows a converse behaviour for a further decrease in $\zeta$ beyond a certain optimum value. Based on the simulation analysis, it is observed that the MSE performance is optimum for $\zeta$ equal to $1 / 5$ times the strongest multipath component in the channel.

\section{CONCLUSION}

A channel model for the implementation of sparse MIMO channels has been proposed. Three SiT compressed channel sensing based estimation techniques has been proposed for frequencyselective time-invariant sparse MIMO communication channels. A thorough analysis based on the simulation results has been presented. The MSE and BER have been used for the performance analysis. Effect of various parameters, such as, the channels' sparsity level, training to information power ratio,threshold coefficient, and message length, has thoroughly been investigated. It has been established that the proposed SiT-CCS, SiT-ThCCS, and SiT-MP techniques outperform the traditional SiT-LS technique for the case of sparse MIMO channels. It has been shown that the proposed SiT-CCS, SiT-MP, and SiT-ThCCS can provided an improvement of $2 \mathrm{~dB}, 3.5 \mathrm{~dB}$, and $5.2 \mathrm{~dB}$ in the MSE at SNR of $12 \mathrm{~dB}$ when compared to SiT-LS in [18], respectively. Consequently, a gain of about $1 \mathrm{~dB}, 2.5 \mathrm{~dB}$, and $3.5 \mathrm{~dB}$ in $\mathrm{SNR}$ has been observed at $\mathrm{BER}=10^{-1.9}$ by the proposed SiT-CCS, SiT-MP, and SiT-ThCCS when compared to SiT-LS [18], respectively. This performance gain in MSE and BER has been observed to increase with an increase in the channels' sparsity.

\section{ACKNOWLEDGEMENT}

The first three authors would like to acknowledge the partial support by EU ATOM-690750 research project approved under the call H2020-MSCA-RISE-2015. The fourth author would like to acknowledge the partial financial support by the National Research Fund, Luxembourg under the CORE project SeMiGod". 


\section{REFERENCES}

[1] B. Mansoor, S. J. Nawaz, B. Amin, S. K. Sharma, and M. N. Patwary. Superimposed training based estimation of sparse mimo channels for emerging wireless networks. In proc. of International Conference on Telecommunications, pages 1-6, May 2016.

[2] S. F. Cotter and B. D. Rao. Sparse channel estimation via matching pursuit with application to equalization. IEEE Transactions on Communications, 50(3):374-377, Mar. 2002.

[3] S. Ariyavisitakul, N. R. Sollenberger, and L. J. Greenstein. Tap-selectable decision-feedback equalization. IEEE Transactions on Communications, 45(12):1497-1500, Dec. 1997.

[4] S. J. Nawaz, K. I. Ahmed, M. N. Patwary, and N. M. Khan. Superimposed training-based compressed sensing of sparse multipath channels. IET Communications, 6(18):3150-3156, Dec. 2012.

[5] J. Ying, J. Zhong, M. Zhao, and Y. Cai. Turbo equalization based on compressive sensing channel estimation in wideband HF systems. In Proc. of International Conference on Wireless Communications Signal Processing, pages 1-5, Oct. 2013.

[6] M. Kocic, D. Brady, and M. Stojanovic. Sparse equalization for real-time digital underwater acoustic communications. In Proc. of OCEANS Conference, 1995.

[7] W. Schreiber. Advanced television systems for terrestrial broadcasting: Some problems and some proposed solutions. Proceedings of the IEEE, 83(6):958-981, Jun. 1995.

[8] I. J. Fevrier, S. B. Gelfand, and M. P. Fitz. Reduced complexity decision feedback equalization for multipath channels with large delay spreads. IEEE Transactions on Communications, 47(6):927-937, Jun. 1999.

[9] J. Zhao, W. Meng, and S. Jia. Sparse underwater acoustic OFDM channel estimation based on superimposed training. Journal of Marine Science and Application, 8(1):65-70, 2009.

[10] G. Gui, A. Mehbodniya, and F. Adachi. Adaptive sparse channel estimation for time-variant MIMO communication systems. In Proc. of IEEE Vehicular Technology Conference, pages 1-5. IEEE, Sep. 2013.

[11] S. F. Cotter and B. D. Rao. Sparse channel estimation via matching pursuit with application to equalization. IEEE Transactions on Communications, 50(3):374-377, 2002.

[12] N. Wang, G. Gui, Z. Zhang, T. Tang, and J. Jiang. A novel sparse channel estimation method for multipath MIMO-OFDM systems. In Proc. of IEEE Vehicular Technology Conference, pages 1-5. IEEE, 2011.

[13] G. Z. Karabulut and A. Yongacoglu. Sparse channel estimation using orthogonal matching pursuit algorithm. In Proc. of IEEE Vehicular Technology Conference, volume 6, pages 3880-3884. IEEE, 2004.

[14] J. Haupt, W. U. Bajwa, G. Raz, and R. Nowak. Toeplitz compressed sensing matrices with applications to sparse channel estimation. IEEE Transactions on Information Theory, 56(11):5862-5875, 2010.

[15] G. Gui, Q. Wan, W. Peng, and F. Adachi. Sparse multipath channel estimation using compressive sampling matching pursuit algorithm. arXiv preprint arXiv:1005.2270, 2010.

[16] D. Han, S. Kim, and J. C. Principe. Sparse channel estimation with regularization method using convolution inequality for entropy. In Proc. of IEEE International Joint Conference on Neural Networks, volume 4, pages 2359-2362. IEEE, 2005.

[17] F. Mazzenga. Channel estimation and equalization for M-QAM transmission with a hidden pilot sequence. IEEE Transactions on Broadcasting, 46(2):170-176, 2000. 
[18] S. He, J. K. Tugnait, and X. Meng. On superimposed training for MIMO channel estimation and symbol detection. IEEE Transactions on Signal Processing, 55(6):3007-3021, 2007.

[19] B. Farhang-Boroujeny. Pilot-based channel identification: proposal for semi-blind identification of communication channels. IET Electronics Letters, 31(13):1044-1046, 1995.

[20] J. K. Tugnait and W. Luo. On channel estimation using superimposed training and first-order statistics. In Proc. of IEEE International Conference on Acoustics, Speech, and Signal Processing, volume 4, pages IV-624. IEEE, 2003.

[21] S. J. Nawaz, M. I. Tiwana, M. N. Patwary, N. M. Khan, M. I. Tiwana, and A. Haseeb. GA based sensing of sparse multipath channels with superimposed training sequence. In Press, Elektronika ir Elektrotechnika.

[22] V. Jungnickel, K. Manolakis, W. Zirwas, B. Panzner, V. Braun, M. Lossow, M. Sternad, R. Apelfrojd, and T. Svensson. The role of small cells, coordinated multipoint, and massive MIMO in 5G. IEEE Communications Magazine, 52(5):44-51, 2014.

[23] Ming Lu, Titus Lo, and J. Litva. A physical spatio-temporal model of multipath propagation channels. In Proc. of IEEE Vehicular Technology Conference, volume 2, pages 810-814, May 1997.

[24] S. J. Nawaz, B. H. Qureshi, and N. M. Khan. A generalized 3-D scattering model for a macrocell environment with a directional antenna at the BS. IEEE Transactions on Vehicular Technology, 59(7):3193-3204, Sep. 2010.

[25] R. Janaswamy. Angle and time of arrival statistics for the gaussian scatter density model. IEEE Transactions on Wireless Communications, 1(3):488-497, Jul. 2002.

[26] K. N. Le. On angle-of-arrival and time-of-arrival statistics of geometric scattering channels. IEEE Transactions on Vehicular Technology, 58(8):4257-4264, Oct. 2009.

[27] N. M. Khan. Modeling and characterization of multipath fading channels in cellular mobile communication systems. Ph.d., Faculty of Engineering, University of New South Wales, 2006.

[28] Y. Barbotin, A. Hormati, S. Rangan, and M. Vetterli. Estimating sparse MIMO channels having common support. In Proc. of IEEE International Conference on Acoustics, Speech, and Signal Processing, pages 2920-2923, May 2011.

[29] E. J. Candes and T. Tao. Decoding by linear programming. IEEE Transactions on Information Theory, 51(12):4203-4215, Dec. 2005.

[30] E. J. Candes, J. Romberg, and T. Tao. Robust uncertainty principles: exact signal reconstruction from highly incomplete frequency information. IEEE Transactions on Information Theory, 52(2):489-509, Feb. 2006.

[31] E.J. Candes and T. Tao. Near-optimal signal recovery from random projections: Universal encoding strategies? IEEE Transactions on Information Theory, 52(12):5406-5425, Dec. 2006.

[32] D. L. Donoho. Compressed sensing. IEEE Transactions on Information Theory, 52(4):1289-1306, Apr. 2006.

[33] W. U. Bajwa, J. Haupt, A. M. Sayeed, and R. Nowak. Compressed channel sensing: A new approach to estimating sparse multipath channels. Proceedings of the IEEE, 98(6):1058-1076, Jun. 2010.

[34] G. Taubock, F. Hlawatsch, D. Eiwen, and H. Rauhut. Compressive estimation of doubly selective channels in multicarrier systems: Leakage effects and sparsity-enhancing processing. IEEE Journal of Selected Topics in Signal Processing, 4(2):255-271, Apr. 2010.

[35] C. R. Berger, Z. Shengli, J. C. Preisig, and P. Willett. Sparse channel estimation for multicarrier underwater acoustic 
communication: From subspace methods to compressed sensing. IEEE Transactions on Signal Processing, 58(3):17081721, Mar. 2010.

[36] E. Candes and T. Tao. The Dantzig selector: Statistical estimation when p is much larger than n. Ann. Statist., 35(6):23132351, Dec. 2007.

[37] S. Chen, A. Livingstone, and L. Hanzo. Minimum bit-error rate design for space-time equalization-based multiuser detection. IEEE Transactions on Communications, 54(5):824-832, 2006.



Bilal Amin received his B.Sc. degree in Electrical Engineering having specialization in Telecommunications from COMSATS Institute of Information Technology, Lahore Campus in 2013 and M.Sc. degree in Electrical Engineering from COMSATS Institute of Information Technology, Islamabad Campus in 2015. He has an experience of more than one year in academia regarding research and teaching. He is currently working as Lecturer with the Department of Electrical Engineering, CIIT Lahore.

His research interest include spread spectrum, channel estimation and equalization, compressed channel sensing, massive MIMO communication systems, signal processing, and adaptive signal processing.

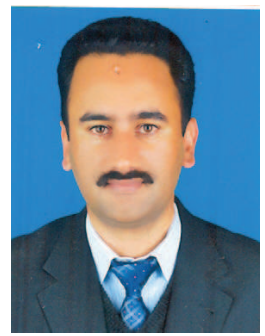

Babar Mansoor received the B.Sc. degree in Computer Engineering from COMSATS Institute of Information Technology (CIIT), Abbottabad, Pakistan, in 2005 and M.Sc. degree in Microelectronic Systems in 2007 from The University of Liverpool, UK. He is currently pursuing his Ph.D. research work in the Department of Electrical Engineering, CIIT, Islamabad, Pakistan. His research areas include wireless communications, signal processing, and MIMO communication systems. 


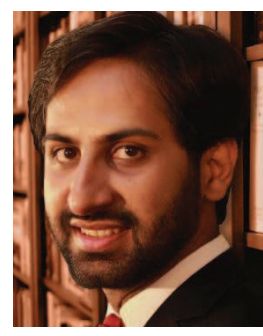

Syed Junaid Nawaz received the B.Sc. and M.Sc. degrees in computer engineering from COMSATS Institute of Information Technology (CIIT) Abbottabad, in Aug. 2005 and Feb. 2008, respectively. He received the Ph.D. degree in electronic engineering from Mohammad Ali Jinnah University Islamabad, in Feb. 2012. Since Sep. 2005, he has worked on several research and teaching positions with CIIT Abbottabad, CIIT Islamabad, Federal Urdu University Pakistan, Staffordshire University UK, and Aristotle University of Thessaloniki Greece. He is currently working as an Assistant Professor with the Department of Electrical Engineering, CIIT Islamabad. He has an active presence in the research community through publications in reputed international journals and conferences in different areas of wireless communications. He is currently working on development of stochastic geometry based solutions for various challenging aspects of emerging $5 \mathrm{G}$ communication networks.

His current research interests include physical channel modeling and characterization, interference modeling, channel estimation and equalization, massive MIMO systems, compressive sensing, vehicle-to-vehicle communications, airborne internet, and adaptive signal processing.

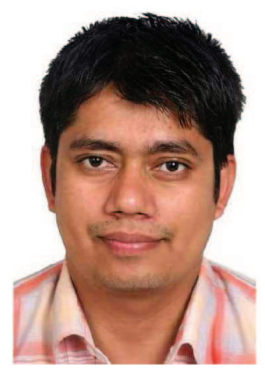

Shree Krishna Sharma received the M.Sc. degree in information and communication engineering from the Institute of Engineering, Pulchowk, Nepal, in 2010; the M.A. degree in economics from Tribhuvan University, Nepal; the M.Res. degree in computing science from Staffordshire University, Staffordshire, U.K., in 2011; and the Ph.D. degree in Wireless Communications from University of Luxembourg, Luxembourg in 2014. Since November 2014, he has been working as a Research Associate in Interdisciplinary Centre for Security, Reliability and Trust (SnT), University of Luxembourg, Luxembourg.

In the past, Dr. Sharma was also involved with Kathmandu University, Dhulikhel, Nepal, as a Teaching Assistant, and he worked as a Part-Time Lecturer for eight engineering colleges in Nepal. He was with Nepal Telecom for more than four years as a Telecom Engineer in the field of information technology and telecommunication. He is the author of more than 50 technical papers in refereed international journals, scientific books, and conferences. He received an Indian Embassy Scholarship for his B.E. study, an Erasmus Mundus Scholarship for his M. Res. study, and an AFR Ph.D. grant from the National Research Fund (FNR) of Luxembourg. He received Best Paper Award in CROWNCOM 2015 conference held in Doha, Qatar and for his Ph.D. thesis, he received FNR award for outstanding PhD Thesis 2015 from FNR, Luxembourg. He has been involved in EU FP7 CoRaSat project, EU H2020 project SANSA, ESA project ASPIM, and Luxembourgish national projects Co2Sat, and SeMIGod. He has been serving as a reviewer for several international journals and conferences, and also as a TPC member for a number of conferences. His research interests include cognitive wireless communications, satellite communications, and signal processing techniques for $5 \mathrm{G}$ and beyond wireless. 


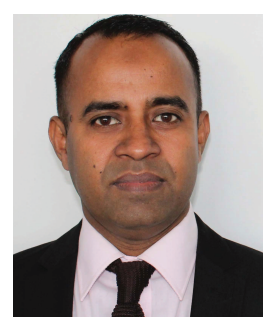

Mohammad Patwary is Professor of Wireless Systems \& Digital Productivity with the Institute for Technological and Environmental Wellbeing and Chair for the Centre of Excellence on Digital Productivity with Connected Services (DiPConS) at Staffordshire University, UK. He received his Ph.D. degree in Telecommunication Engineering from The University of New South Wales, Sydney, Australia in 2005 and BEng(Hons). in Electrical and Electronic Engineering from Chittagong University of Engineering and Technology, Bangladesh in 1998. He was with the General Electric Company of Bangladesh from 1998 to 2000 and with Southern-Poro Communications, Sydney Australia from 2001-2002 as R \& D Engineer. He worked at the University of New South Wales, Sydney Australia as Lecturer from 2005-2006; then at Staffordshire University, UK as Senior Lecturer from 2006-2010. Current research interests of DiPConS include future generation of cellular network architecture; signal detection, estimation and optimisation for future generation of wireless receivers; joint source-channel characterisation for wireless sensor networks (Heterogeneous Networks \& IoT) and spatial diversity schemes for wireless communications. 